ISSN: $1782-2041$

DOI: $10.25518 / 1782-2041.1181$

\title{
L'Intelligibilité de la pratique, après coup
}

\author{
Par HERVÉ OULC'HEN
}

Université de Liège

Le jeudi 26 avril 2018, durant la troisième journée du douzième séminaire annuel du Centre de recherches phénoménologiques, une table ronde fut consacrée à l'ouvrage d'Hervé Oulc'hen, L'Intelligibilité de la pratique. Althusser, Foucault, Sartre. Paru en 2017 aux Presses Universitaires de Liège, ce travail fut présenté par Florence Caeymaex avant d'être discuté par Gildas Salmon, Chiara Collamati, Soheil Ghanbari et Ferhat Taylan - que nous remercions chaleureusement d'avoir participé à cette rencontre scientifique.

Ci-dessous, le lecteur trouvera d'abord les questions posées sur le livre et, ensuite, les réponses données par l'auteur. Les points soulevés par Gildas Salmon font l'objet d'une explication dans les sections 1 et 2 du texte d'Hervé Oulc'hen. Les parties 2 et 3 répondent quant à elles aux interrogations formulées par Chiara Collamati et Soheil Ghanbari. Enfin, les remarques de Ferhat Taylan sont examinées dans la section 4 qui conclut ces échanges autour du problème de l'intelligibilité de la pratique. Si ces discussions conduisirent Hervé Oulc'hen à préciser l'articulation, les sources et les grilles d'interprétation de son livre, elles l'amenèrent également à revenir sur ces trois problèmes: les rapports épistémiques entre sciences sociales et philosophie, d'une part, les enjeux politiques du concept d' «institution », d'autre part, et, enfin, le destin des concepts de vérité et de dialectique dans les philosophies de la pratique qui furent développées par la troïka Althusser-Foucault-Sartre.

\section{Questions et remarques de Gildas Salmon (CNRS, LIER-FYT)}

Mes questions porteront pour l'essentiel sur la construction d'ensemble de l'ouvrage. Ma principale interrogation porte sur l'articulation des différentes 
parties du texte. Y a-t-il véritablement un problème commun à tous ces auteurs, ou le terme de «pratique » recouvre-t-il plusieurs problématiques distinctes chez Althusser, Foucault et Sartre ? Je me demande si le problème de l'intelligibilité de la pratique au sens strict, c'est-à-dire entendu comme ressaisie théorique d'une activité conçue sur le modèle de la production, ne se limite pas à Althusser (et dans une certaine mesure Bourdieu) ? Il me semble n'être compréhensible qu'à partir du croisement très singulier de Marx et de Bachelard, c'est-à-dire d'une double survalorisation de la théorie pure et de la pratique comme production. En un sens, j'ai l'impression que la conclusion de l'étude d'Hervé aboutit à une impasse, et que c'est plutôt dans le contournement de ce problème, ou dans sa dissolution, que se produisent les véritables avancées, notamment dans l'analyse des savoirs. Je m'interroge en particulier sur l'articulation des chapitres 2 et 3 (le passage aux pratiques de soi chez Foucault à partir d'Althusser, qui me semble recouvrir un véritable saut en dépit du Machiavel d'Althusser), et aussi sur le retournement qui est opéré dans le dernier chapitre où ce n'est plus l'intelligibilité de la pratique, mais celle de la vérité (à partir des pratiques) qui fait problème. Ces décalages sont explicités dans le livre, mais j'aimerais qu'Hervé revienne sur la perspective d'ensemble : s'il y a une unité problématique sous-jacente (ce qui est affirmé dans l'introduction), comment la formuler? Si l'enjeu est plutôt de cartographier une dispersion des usages de la pratique, comment caractériser l' « espace de dispersion » ainsi défini ? Doit-on conclure que le problème de l'intelligibilité de la pratique au sens strict (althussérien) est en définitive un problème mal posé, ou sinon comment comprendre la relative impasse à laquelle il aboutit ?

Une deuxième question, corrélative, porte sur la méthode : dans chaque chapitre, l'auteur précédent sert à analyser le suivant : Foucault est lu à partir d'Althusser, Sartre à partir de Foucault, et, dans le dernier chapitre, on pourrait dire que Foucault est lu à partir de Sartre. J'aimerais qu'Hervé précise les enjeux de sa méthode de lecture singulière qui fait ressortir la cohérence de chaque auteur en l'interrogeant pour ainsi dire de biais. Cette question est liée à la précédente en ce qu'il s'agit là aussi de le conduire à préciser le type d'unité ou de décalage qu'il veut faire apparaître.

Dans la suite de ma première question, j'interrogerai aussi Hervé sur l'absence de Bourdieu, qui, pour être évoqué plusieurs fois, n'est pas traité pour lui-même dans le livre, alors qu'il semble être celui qui entre le mieux dans la problématique de l'intelligibilité de la pratique telle qu'elle est définie à partir d'Althusser, et qui parvient en quelque sorte à opérationnaliser ce questionnement dans un savoir sociologique. Pourquoi ne pas avoir intégré plus directement la sociologie bourdieusienne? 
Enfin, une question plus générale portera sur la place spécifique des sciences sociales dans cette configuration. L'impression d'ensemble qui se dégage de l'ouvrage est que les références à Marx d'une part, et à Nietzsche de l'autre, sont plus importantes que le développement des sciences sociales de l'époque. Jusqu'où Hervé serait-il prêt à adopter la thèse d'une forme de clôture de la philosophie sur elle-même (puisque Marx est intégré à la tradition philosophique) dans un moment où on proclame l'avènement des sciences humaines ? Cette question se pose en particulier dans le dernier chapitre, où la référence à Nietzsche semble écraser la ligne plus durkheimienne qu'on trouve notamment chez Gernet. Or, ceci rejaillit sur le concept de pratique ; car, dans la généalogie nietzschéenne, il s'agit de la lutte entre des évaluations et des types d'hommes hétérogènes, plutôt que des effets de pratiques collectives productrices de normes. Comment les deux choses s'articulent-elles à la fois chez Sartre et chez Foucault? Dans quelle mesure leur généalogie de l'idée de vérité est-elle transformée par le développement des sciences sociales?

\section{Questions et remarques de Chiara Collamati (Université de Liège)}

J'aborderai ton livre par la fin, en commençant par les ouvertures conclusives, pour remonter à quelques-uns des points cruciaux de ton argumentation. $\mathrm{Au}$ bout de ce parcours magistralement tracé sur le terrain d'un problème commun à Althusser, Foucault et Sartre, celui justement de l'intelligibilité de la pratique et des théories des ensembles pratiques, le livre démontre la nécessité d'une altération épistémologique réciproque entre la philosophie et les sciences sociales, à partir de laquelle pourrait s'esquisser une autre archéologie que celle de Foucault (p. 382). Cette nouvelle archéologie devrait se caractériser par une lecture historico-conceptuelle des sciences sociales, qui serait capable d'assumer l'irréductibilité du hiatus existant entre sens objectif et sens subjectif des pratiques, de valoriser le désajustement entre habitus et règles, mais aussi de rendre productive la désadaptation dans l'adaptation. Or, pour n'être qu'une allusion, l'expression " histoire conceptuelle " ${ }^{1}$ me semble néanmoins décisive, car elle indique une pratique de la philosophie qui devrait être capable de

${ }^{1}$ Cette expression désigne en réalité une méthode historiographique et philosophique bien précise - la même qui inspire, à certains égards, la recherche de Karsenti à laquelle tu te réfères positivement à plusieurs reprises. L'histoire conceptuelle dans sa formulation allemande (Koselleck, Brunner) considère les concepts comme des formations constituées par plusieurs couches, c'est-à-dire par un ensemble de signifiants qui dérivent chronologiquement de temporalités différentes. Se définissant par sa propre stratification, le concept est lui-même historique, en ce qu'il est l'outil produit 
sortir et de la praxis comme foyer privilégié de donation du sens et des théories extra-anthropologiques de l'origine du sens (selon les mots de Sebag que tu reprends). Autrement dit, chez Sartre et Foucault, cette philosophie devrait être capable d'aller un peu plus loin. Dans ce jeu d'altération réciproque avec les sciences sociales, elle devrait devenir une histoire conceptuelle, une histoire des concepts, où le génitif n'est plus objectif mais subjectif : le concept comme lieu propre de la pratique philosophique, dans son double effort pour comprendre la genèse du concept de concept, ainsi que le rapport de celui-ci à la pratique.

Ton livre nous montre l'importance de penser la pratique, et donc aussi la pratique politique, au-delà de sa réduction à une théorie. On peut préciser cela en disant que la question devient celle de penser la politique en dehors de la science politique moderne telle que s'est affirmée à partir du $17^{\mathrm{e}}$ siècle. À l'intérieur de celle-ci, la pratique est une champ d'application de la théorie ; l'agir politique s'affirme comme un champ de savoir autonome et rigoureusement fondé ; la pratique n'est plus l'exercice de la vertu dans la contingence d'une situation, mais elle devient le résultat nécessaire d'un calcul logique. Au $17^{\mathrm{e}}$ siècle se produit une rupture qui marque le seuil de scientifisation de la politique, celle-ci devenant le lieu d'application de principes dont la validité dérive de la démarche scientifique qui les a produits. Cela implique aussi une transformation de la structure épistémique du savoir pratique ; laquelle transformation n'est pas tant une transition depuis la notion de vertu (Aristote ou Machiavel) vers celle de science (Hobbes ou Pufendorf), car il s'agit plutôt d'un déplacement qui est opéré depuis une science de la vertu vers une science théorique du droit. Voici donc ma première question : reconnaitre, comme tu le suggères, la possibilité d'une approche historico-conceptuelle des sciences sociales n'impliquerait-il pas, en même temps, de produire une pensée philosophique-politique qui puisse garder l'effort épistémique de conceptualisation, tout en montrant les limites et de la science politique moderne et de la science de l'histoire qui la soutient?

Cette question de la conceptualisation est au cœur des critiques que tu adresses à Althusser (dont l'absence dans la couverture du livre fait signe, peut-être, vers quelque chose de plus que la contingence historique de la fameuse photo !) et qui touchent principalement la priorité assignée au plan théorique qui se révèle dépourvu d'un ancrage véritable dans ses conditions réelles

et utilisé par la science de l'histoire dans la compréhension et l'exposition de son propre objet. C'est pourquoi le temps spécifiquement historique, celui qui fait l'objet de la science de l'histoire (Historik), est un temps défini par la « contemporanéité du non contemporain ». 
(p. 179). La confrontation critique que tu mènes avec Althusser fait souvent de ce dernier le point-limite d'une idée de la pratique qui ne peut devenir opérationnelle qu'à travers l'interaction construite entre un "Sartre post-foucaldien » et un Foucault qu'on découvre finalement plus sartrien qu'on ne l'imaginait. Or, tu ne manques pas de souligner que, chez Foucault par exemple, l'enjeu n'est jamais celui de considérer le savoir comme une forme de la pratique (et inversement : " pour Foucault la pratique n'est pas le critère pertinent pour la production du savoir », p. 235). Cela conduirait en effet à considérer l'élaboration des connaissances comme une pratique sociale parmi d'autres ; comme le simple reflet des pratiques sociales. L'enjeu est plutôt de maintenir une certaine autonomie à l'ordre du savoir, dans ses modalités d'articulation (et non pas de réduction) à d'autres pratiques non discursives, sans passer par l'hypothèse d'une expérience pratico-sociale fondatrice, unificatrice, commune, première, etc. Mais le problème surgit, à mes yeux, quand tu essayes de sortir de cette impasse en distinguant chez Sartre les termes de concept et de notion, mais aussi en valorisant la priorité pratique de la totalisation immédiate (ou « incarnation ») comme mode d'intelligibilité des « pratiques ici et maintenant $»$ (p. 359 sq.).

J'en arrive, dès lors, à ma deuxième question. L'opposition entre concept et notion n'est pas formulée de manière précise et exhaustive par Sartre. Ce dernier la mentionne dans l'entretien paru dans L'Arc en 1966, et il y dédie quelques lignes dans la Critique de la Raison dialectique. Or il me semble que, pour pouvoir le suivre comme tu le proposes, nous devrions d'abord nous demander quelle est la définition du concept qui est en jeu dans sa philosophie. Tu suggères qu'il s'agit d'une vision kantienne du concept (p. 210); mais cela ne risque-t-il pas d'affaiblir la force euristique du rapport dialectique entre le réel et la pensée, que Sartre construit au travers d'une assimilation critique de Hegel et de Marx ? En saluant le refus sartrien de produire un concept du temps historique (le temps ne pouvant être l'objet d'un concept en raison de la clôture figée qui est produite par toute conceptualisation), tout en le faisant jouer contre la tentative althussérienne de définir l'objet théorique du matérialisme historique, ne risque-t-on pas d'affaiblir la spécificité de la pratique philosophique ? La Critique de la raison dialectique, tout en étant une expérience critique que n'importe qui peut conduire, a été quand même écrite par Sartre au prix de nombreux efforts, et à travers la construction de concepts bien définis.

En quoi la notion serait-elle plus adéquate au fonctionnement du statut épistémique des sciences sociales? Du point de vue qui est le tien, quelles implications y aurait-il à considérer la société et la démocratie comme des con- 
cepts et non des notions ? Serais-tu d'accord de dire que la notion fonctionnerait comme l'horizon ou le champ au sein duquel une certaine constellation de concepts peut devenir opérationnelle?

Ta valorisation de ce que Sartre nomme incarnation ou totalisation immédiate me permet aussi d'ajouter une troisième question liée à la précédente. Aux pages 273-274 de ton livre, tu montres comment le primat sartrien de la praxis (qui s'atteste non seulement sur le plan ontologique et moral, mais aussi, et d'abord, sur le plan épistémologique), fait de la totalisation en cours la seule démarche capable de ressaisir une situation historique donnée, comme si elle était encore présente (p. 269) ; ce qui éloigne Sartre de la notion foucaldienne d'archive et du primat épistémologique du passé qui la soutient, mais aussi de la conception althussérienne de la connaissance (p. 316), car, chez Sartre, les idées émergeant de la praxis, elles ont constitutivement une implication situationnelle et une utilité pratique. La praxis se donnant ses propres lumières, c'est l'idée pratique qui nous sert à nous expliquer notre propre situation.

Cette démonstration te permet de ranger Sartre du côté de l'historiographie du présent, qui est pratiquée par Marx dans ses écrits sur la France, et d'analyser une certaine manière de se situer dans l'époque, et, ainsi, de penser sur un mode problématique le présent dans lequel on vit et on agit. La manière dont tu présentes la pensée de Sartre comme « critique en situation » permet dès lors de déplacer le double reproche que Foucault formule contre lui : celui d'être un homme en retard sur son époque (p. 216) et d'être un homme qui colle tellement à l'épistème de son époque qu'il ne peut réellement la saisir de manière critique (p. 253). Ainsi ta lecture démontre quelque chose que Sartre avait pressenti dans Les Mots : le retard qu'il a sur son époque est exactement ce qui lui fait gagner de l'avance sur ses contemporains. Ce phénomène de retard revient à plusieurs reprises dans ton livre à travers la catégorie bourdieusienne d'hystérésis. Je voudrais te demander s'il te semble pertinent de sortir cette notion (qui décrit au départ un phénomène de la science physique) du domaine sociologique (où elle a été souvent utilisée pour désigner une décalage entre le plan des nécessités structurales et celui de l'incorporation effective des pratiques) pour repenser le temps historique. Autrement dit, peut-on considérer la compréhension de la temporalité proposée par la sociologie comme une intervention épistémique dans les théories modernes de l'histoire ?

Je termine par une quatrième question (articulée en trois sous-questions) qui me permet de souligner la force des analyses déployées par ton livre autour des « politiques de la vérité », à travers un dialogue entre le jeune Sartre et le Foucault des années septante et quatre-vingt. $1^{\circ}$ Peux-tu préciser en quoi la manière dont Sartre construit une " histoire matérialiste de l'idée de vérité » (p. 324), en faisant de celle-ci non seulement un effet des luttes, mais aussi un 
rapport pratique présupposant, à la fois, un procès de travail entre les hommes et la matière, et un procès d'échange (p. 317), en quoi cette manière diffère de la vision althussérienne que tu critiques justement à cause de «l'importance accordée au procès du travail comme modèle du procès de connaissance » (p. 191) ? $2^{\circ}$ Dans l'architecture de ton argumentation et dans le geste herméneutique que tu accomplis au chapitre 5, quelle place pourrait trouver la réflexion sartrienne des années 1960 autour de l'éthique et du normatif en tant que disposition pratique pensée justement sur un plan pré et extra-juridique ? $3^{\circ}$ Dans ta lecture, la méthode du « comme si », méthode de l'histoire-fiction chez Foucault et de l'histoire-légende chez Sartre, permet d'approcher la vérité comme une dimension immanente aux pratiques, et ce, sans présupposer aucun universel à son sujet, ni la décomposer en ses éléments constitutifs (p. 342); en outre, elle permet de poser à nouveaux frais le problème de l'idéologie, qui cesse d'emblée d'être la fausse représentation d'un contenu. On peut valoriser cette méthode en remontant à l'étymologie latine de fingere, qui renvoie seulement de manière indirecte à la simulation, à l'imitation comme contrefaçon, car, en premier lieu, ce verbe indique une créativité matérielle : le fait de modeler, de donner une forme à de la cire ou de l'argile. De la même manière, dans le lexique du droit, la fictio est la technique par laquelle on peut attribuer la personnalité juridique à une entité dépourvue de consistance ontologique prénormative. Dans les deux cas, la fiction désigne une trans-formation, une action par laquelle quelque chose intervient sur quelque chose d'autre et le modifie, conférant ainsi une forme à ce qui n'en avait pas avant. Cependant, il me semble que « fictionner l'histoire » c'est aussi, d'une certaine manière, la représenter. Certes, il ne s'agit pas pour Sartre et Foucault de représenter la vérité, ni de reproduire la prétention d'un système de concepts à subsumer la réalité en la représentant. Mais la conséquence nécessaire de cette position n'est-elle pas d'affirmer que la philosophie se trouve déjà dans les pratiques, ou mieux dans les narrations fictionnelles des pratiques ? Dans ce cas, comment soustraire la méthode historico-fictionnelle au péril «postmoderniste » de la prolifération des narrations mythopoiétiques ? Si une histoire fictionnelle des régimes modernes de vérité doit reconstruire leurs ancrages dans des pratiques archaïques et rituelles, ne risque-t-elle pas de produire des effets de projection rétroactive qui seraient justement insoutenables du point de vue d'une histoire-conceptuelle? 


\section{Questions et remarques de Soheil Ghanbari (FNRS, Université de Liège)}

La Critique de la Raison dialectique ressaisit l'intelligibilité de la pratique à partir de «notions » et de « concepts» problématiques. Comme l'a en effet rappelé, votre livre montre que la signification de ce vocabulaire n'est pas claire. Je souhaiterai que vous reveniez plus longuement sur ce point. Que veulent dire ces termes? Et surtout, quelle conception de la dialectique impliquent-ils ? En ressaisissant la pratique par des notions ou des concepts, Sartre ne retombe-t-il pas dans une « dialectique de l'entendement » qu'il voulait justement éviter?

\section{Questions et remarques de Ferhat Taylan (FNRS, Université de Liège)}

Il me semble qu'un des grands mérites du livre consiste précisément à déjouer d'emblée, dès la position du problème, la séduction qu'exerce « le primat de la pratique » surtout dans la lignée althussérienne : ce " primat », comme tu le montres, est surtout une manière d'unifier la pluralité des pratiques, de s'emparer ainsi de leur prestige et, finalement, une tentative de faire descendre les structures (déguisées en praxis) dans la rue, sous l'autorité du théoricien luimême déguisé en praticien. On voit bien que Foucault, parce qu'il n'érige pas la pratique en pierre de touche et s'appuie sur la pluralité des pratiques dans la formation des discours, permet d'appuyer cette opération de démystification « du primat de la pratique ». Or, à demeurer dans cette confrontation Althusser/Foucault, on se situe sur une ligne de crête entre deux types de réflexivité qui prennent chacun en charge l'intelligibilité des pratiques : le premier essentialise « la pratique comme opérateur d'explication des pratiques », opérateur qui permet de redonner à la philosophie un certain prestige dès lors qu'elle se définit comme la pratique théorique ; le second, au contraire, propose de suivre les logiques propres aux pratiques, quitte à être reconduit à l'histoire et aux sciences sociales comme régimes de connaissance de ce que tu appelles « le divers des pratiques » (p. 18). L'intérêt de cette dernière expression vient du fait qu'elle fait apercevoir un déplacement majeur opéré au sein même de la philosophie qui, au-delà de trilogie Sartre-Althusser-Foucault, se trouve non plus face au défi de synthétiser la connaissance du divers du sensible, mais sommée de se rendre compte du divers des pratiques et de leur intelligibilité. Que ce problème se produise à l'intérieur de la philosophie pour induire une crise de ses frontières avec les sciences sociales et l'histoire, c'est ce que montre l'ouvrage - à cet égard, la conclusion (p. 379) est tout à fait éclairante 
quant à l'actualité de l'opposition structure/pratique qui apparaît comme un partie du programme des sciences sociales.

Au-delà de son intérêt majeur et évident pour les sciences sociales et l'histoire de la philosophie, le livre déploie une dimension plus spécifiquement politique concernant la portée « transformatrice » de la praxis ou des pratiques revendiquées par les penseurs que tu examines et que j'aimerais discuter à partir de la problématique de l'institution des pratiques (que tu abordes par le trio Althusser-Sartre-Foucault). On le sait, toute une lignée de philosophes politiques s'y sont intéressés à peu près au même moment : soit sous la forme d'une interrogation critique concernant la bureaucratie, notamment soviétique (avec comme corrélat la question de savoir comment les pratiques révolutionnaires pourraient ne pas tomber dans une réification institutionnelle du Parti et de l'État); soit sous la forme plus positive d'une recherche sur l'institution de la société ou d'un groupe par la stabilisation de ses propres pratiques (Castoriadis, mais aussi la lignée de la psychanalyse institutionnelle). Interroger la tension entre institution et pratiques serait peut-être une autre manière d'aborder la tension entre pratiques et structures, d'autant plus qu'un rapprochement est signalé avec la philosophie sociale (p. 380). La question est de savoir ce qui se passerait, au niveau de la configuration théorique que tu dessines, si on transposait le duo « structure/pratiques » en « institution/pratiques », en mobilisant du même coup non seulement l'intelligibilité des pratiques mais aussi leur dimension transformatrice par leur institutionnalisation même.

On voit bien à quel point la réflexion de Sartre sur le devenir inerte des pratiques en institution est centrale dans ces débats : si l'institution « a ce caractère contradictoire et souvent signalé par les sociologues d'être une praxis et une chose » (J.-P. Sartre, Critique de la raison dialectique, Gallimard, Paris, 1960, p. 687), il convient de tenter d'empêcher qu'elle se définisse progressivement comme une essence, en définissant aussi les hommes comme des moyens inessentiels de la perpétuer. L'une des contributions les plus importantes de Sartre à l'intelligibilité des pratiques apparaît peut-être dans cette réflexion sur la possibilité d'une institution non-réifiante des pratiques, et ce, précisément, parce qu'elle paraît absente, pour des raisons différentes, chez Althusser comme chez Foucault. D'une part, il est évident que le problème de l'institution des pratiques n'est pas celui de leur intelligibilité : il n'est pas seulement question de se référer à « ce que font les hommes et à la manière dont ils le font » comme le dit Foucault, ni d'examiner l'intentionnalité d'une praxis qui est impliquée par le pratico-inerte sartrien (p. 233) et son devenir. De ce point de vue, on voit s'opérer un retournement - en tous cas c'est une conséquence possible du chapitre 4 de ton livre : en voulant échapper, par ce qu'elle appelle le «théorico-actif », à la conception sartrienne de la pratico-inertie et 
à la question de la « libre praxis sous le poids aliénant des institutions » (p. 234), l'archéologie foucaldienne nous laisse dans la région indécise et contingente de «l'institution pure de l'ordre». Certes, la finalité généalogique du projet foucaldien entendue comme « point d'ancrage pour la pratique et l'invention des possibles » indique un horizon d'action dans cette contingence de l'ordre, mais, et ce n'est pas une révélation, cette finalité généalogique ne conduit pas Foucault à une réflexion "positive » sur l'institution des pratiques disciplinaires ou « juridico-discursives ».

Toutefois, la confrontation de Foucault avec Sartre, ainsi que tu l'opères sur le terrain des pratiques qui sont abordées du point de vue de leur institution, permettrait peut-être d'aller au-delà du simple constat (que l'on fait souvent, même si tu ne le fais pas) de l'absence d'une dimension normative des pratiques collectives chez Foucault. Il y a sans doute un dénominateur commun entre les auteurs abordés : ce n'est pas n'importe quelle pratique, mais les pratiques répétées parce que répétables qui sont particulièrement productives d'effets sociaux ou politiques sur la longue durée. À cet égard, comme tu l'indiques aux pages 313 et suivantes de ton livre, Le Sens pratique de Bourdieu parait plus salutaire que les outils foucaldiens. Or, chez Foucault, qui sur ce point suit peut-être Althusser, ces pratiques répétées ou régulières sont surtout celles de disciplines qui font advenir des subjectivités, tout comme les institutions pénitentiaires ou asilaires produisent des corps dociles et des subjectivités obéissantes. Le sujet est toujours institué, mais jamais instituant, sauf lorsqu'il s'auto-institue à travers des pratiques de subjectivation, qui sont abordées par Foucault dans la dernière période de ses travaux (ascèse, pratiques de soi, etc.). La question est alors de savoir si « l'horizon éthopoiétique» du dernier Foucault se penchant sur l'invention des formes de subjectivité dans les pratiques peut être vu comme une manière d'aborder - enfin, et de manière avant tout subjective et intersubjective, mais pas vraiment collective - la question de l'institution de pratiques autres que celles disciplinaires ou imposées. Or, s'il est vrai rétrospectivement que nous ne sommes pas sortis de la question de l'institution des pratiques collectives « alternatives », il se pose aussi la question de savoir si lire Foucault avec Sartre permettrait de penser «l'horizon éthopoiétique » comme celui d'une institution des pratiques collectives. Plutôt que de commenter ton beau livre, je voudrais donc t'interroger sur les possibilités qu'il ouvre quant aux « pratiques que l'on souhaiterait répéter $\gg$. 


\section{Réponses d'Hervé Oulc'hen (Université de Liège)}

Ce texte constitue la version remaniée de mes réponses à la table-ronde qui s'est tenue le 26 avril 2018 à l'Université de Liège autour de mon ouvrage, L'Intelligibilité de la pratique. Althusser Foucault, Sartre, paru en 2017 aux Presses Universitaires de Liège. Outre l'Unité de recherche Phénoménologies de Liège qui a rendu possible l'organisation matérielle de cette journée, je tiens à remercier très vivement Fabio Recchia, qui en est l'instigateur, ainsi que les différents intervenants lors de la table-ronde : Florence Caeymaex d'une part, pour la belle présentation qu'elle a faite de mon ouvrage, Chiara Collamati, Soheil Ghanbari, Gildas Salmon et Ferhat Taylan, d'autre part, pour les questions riches et percutantes qu'elle et ils m'ont adressées. Pour une meilleure fluidité du propos, et pour éviter d'éventuelles redites, j'ai choisi de regrouper mes réponses autour de quelques thèmes que j'ai pu voir émerger à travers les différentes questions.

\section{Le «primat de la pratique » : retour sur l'équivocité d'un maître-mot}

Comme le souligne Ferhat Taylan, l'une des motivations premières de mon livre était de déjouer l'effet de prestige alloué au maître-mot d'un " primat de la pratique ", fort en vogue dans la séquence intellectuelle française des années 60 qui m'a intéressée. Dans son séminaire de 1975-1976 récemment publié sous le titre Théorie et pratique, Jacques Derrida déconstruit de manière magistrale les significations attachées à l'articulation de la théorie et de la pratique. Tout regard théorique, dit-il, est précédé d'un «ça me regarde » qui constitue le point aveugle de la théorie elle-même, et qui la constitue « depuis une instance pratique $»^{1}$. La théorie, tournée vers l'extérieur, est aveugle à l'injonction pratique qui la motive de l'intérieur, et que Derrida exprime d'une manière très prosaïque : "Faut le faire ». Les trois auteurs que j'ai convoqués dans le livre, Althusser, Foucault, Sartre, se sont attelés à cette question, en essayant de rendre intelligible ce primat de la pratique (« faut le faire »), caché jusque dans le théorique.

Pour répondre d'emblée à la question posée par Gildas Salmon sur ma grille de lecture, je dirai donc que ce sont les difficultés et les apories afférentes à l'idée de primat de la pratique qui m'ont amenées à proposer une lecture indirecte, de biais, de chacun des trois auteurs à la lumière d'un des deux

${ }^{1}$ J. Derrida, Théorie et pratique. Cours de l'ENS-Ulm 1975-1976, Paris, Galilée, 2017, p. 36. 
autres. Plutôt que de proposer une lecture purement internaliste de l'œuvre de chaque auteur dans l'espoir d'y trouver une théorie systématique de la pratique $^{1}, \mathrm{j}^{\prime}$ ai préféré, comme je m'en explique dans l'introduction, privilégier des thèses et des énoncés intempestifs articulés autour de ce problème, hérité de Marx, d'un primat de la pratique. J'ai fait le pari que le dialogue ainsi reconstruit entre des auteurs qui, historiquement, n'ont pas réellement débattu ensemble sur le fond - sinon sur le mode du sarcasme (Sartre et Foucault, Sartre et Althusser) ou de l'éloge ponctuel (Althusser et Foucault) — peut nous délivrer de la querelle de l'humanisme, dont les effets sclérosants se font encore sentir aujourd'hui chez de nombreux foucaldiens et althussériens, qui répètent à l'envi que Sartre n'a rien à nous apprendre. De manière structurale, j'ai envisagé les thèses des auteurs dans leur valeur différentielle, pour la construction du problème de l'intelligibilité de la pratique.

Si Althusser occupe une place prépondérante dans l'ouvrage, c'est parce qu'il a beaucoup fait pour démystifier toute lecture subjectiviste et historiciste du primat de la pratique (qu'il voyait à l'œuvre dans les philosophies de la praxis : principalement chez Gramsci et Sartre), en montrant qu'il demeure indissociable de son autre, le théorique. D'où son idée, développée dès 1965 dans Pour Marx et Lire Le Capital, d'une systématisation théorique des pratiques sous un modèle univoque - la production - emprunté au concept marxien de procès de travail (combinant les quatre éléments que sont l'objet, l'instrument, l'activité et le produit du travail). La pratique comme production est bien "l'opérateur d'explicitation des pratiques », mais paradoxalement, c'est moins la production matérielle que la production théorique, pensée par analogie avec le travail, qui constitue cet opérateur. Je m'accorde avec Gildas Salmon pour dire qu'on assiste, avec Althusser, à un « croisement très singulier de Marx et de Bachelard » consistant à survaloriser et le pratique pur, et le théorique pur. Mais, comme je m'en explique à la page 100 du livre, c'est d'abord le croisement entre Marx et Spinoza qui permet de rendre compte de cette double survalorisation. Et c'est ce croisement qui explique par ailleurs la place privilégiée qu'Althusser accorde à la « pratique théorique » dans sa systématisation des pratiques, dont le pluriel couvre des strates aussi diverses que le travail, l'idéologie, la politique et l'art. Cette systématisation a un prix, qui consiste à autonomiser le travail du théorique hors des pratiques au sens large - on reconnaitra la fameuse " coupure épistémologique ». C'est dans la pratique spécifique des sciences, et dans la manière dont les sciences écrivent leur propre histoire, que l'on peut voir le théorique à l'œuvre : le théorique comme production de concepts scientifiques d'un côté, et comme épistémologie de

${ }^{1}$ Bien qu'une telle systématicité existe chez Althusser — j’y viens plus loin. 
l'autre - sous la forme réflexive de la «théorie de la pratique théorique », qu'Althusser pense à l'aide du modèle spinoziste de l'idée de l'idée.

Il est certain que, jusqu'à un certain point, Foucault revendique cet héritage althussérien, qu'il situe plus largement dans ce qu'il appelle la tradition française d'épistémologie historique et de philosophie du concept (par opposition aux philosophies de la conscience). Mais s'il se sépare nettement d'Althusser, c'est dans sa manière d'aborder le « divers des pratiques » : il s'agit pour Foucault de traiter cette diversité pour elle-même, sans l'inféoder à un modèle théorique homogénéisant qui ne fait finalement que dramatiser encore plus le primat de la pratique, en différant sans cesse, dans la systématisation théorique, le moment constituant du passage à la pratique. Nul besoin pour Foucault d'invoquer un primat de « la » pratique sur la théorie ni, comme Althusser, de construire le concept théorique d'un tel primat, puisque les pratiques sont toujours déjà traversées par des régimes de savoir et de discours, dans lesquelles elles se réfléchissent en se donnant à elles-mêmes leurs propres lumières (pour reprendre une expression qui n'est pas de Foucault mais de Sartre). On assiste alors avec Foucault à un déplacement significatif du pratico-théorique au pratico-discursif : le théorique au sens fort où l'entend Althusser n'a plus de privilège spécifique puisqu'il apparaît comme une pratique discursive parmi d'autres. Pour répondre à la question de Gildas Salmon concernant l'articulation des chapitres 2 ( «Avec Althusser : les instances de la pratique ») et 3 ( Foucault, philosophie pratique ») du livre, je dirais qu'il y a bien, à ce niveau, un complet renversement dans la notion même d'intelligibilité de la pratique, puisque cette intelligibilité n'est plus fournie par l'autonomie du théorique, elle est reversée dans l'éclatement des discours immanents aux pratiques.

\section{Penser aux frontières ${ }^{1}$ : la philosophie à l'épreuve des sciences sociales}

Ce déplacement de la pratique théorique à la pratique discursive se traduit, comme le souligne Ferhat Taylan, par une « crise de frontières » entre la philosophie et son dehors, à savoir les discours qui se sont efforcés de rendre raison du divers empirique des pratiques: en l'occurrence, l'histoire et les sciences sociales. Penser aux frontières : Althusser s'en est quant à lui bien

\footnotetext{
${ }^{1}$ Je fais ici référence au titre du recueil de poèmes parlés de David Antin, Parler aux frontières [1974], Paris, Vies Parallèles, 2017. Antin brouille délibérément les frontières entre divers modes de discours dans ses improvisations (poésie, mythes, récits ethnologiques, philosophie, critique artistique et littéraire, etc.).
} 
gardé, focalisé qu'il était sur la nécessité de préserver l'autonomie du théorique de toute compromission avec les sciences sociales - c'est l'objet de la critique d'E.P. Thompson dans The Poverty of Theory, que j'analyse dans le livre aux pages 205-212. Il y a pourtant bien chez Althusser une tentative de mise en crise du discours philosophique. Comme le souligne Derrida, chez Althusser « le philosophique comparaît devant le théorique et constitue une région du théorique $»^{1}$. Mais qu'est-ce donc que ce théorique ainsi érigé en tribunal, si ce n'est une autre manière de nommer le philosophique ? C'est précisément ce tour de passe-passe kantien qui ne s'avoue pas comme tel, cette tentative avortée de mise en crise du discours philosophique, que Foucault diagnostique au début des Leçons sur la volonté de savoir, où Althusser est implicitement visé à travers la critique du modèle spinoziste de l'idée vraie, ainsi que je le montre aux pages 183-191 du livre.

La crise de frontières est comme appelée par le problème de l'intelligibilité de la pratique. Mais n'est-on pas amenés, avec des auteurs tels qu'Althusser, Foucault et Sartre, à refermer le discours philosophique sur lui-même, comme le suggère Gildas Salmon ? Je montre dans le dernier chapitre, « Politiques de la vérité ", que la double référence à Marx et à Nietzsche permet à Foucault d'ordonner sa généalogie à un modèle agonistique, ce qui a pour effet d'écraser le gain d'intelligibilité des paradigmes propres aux sciences sociales qu'il mobilise par ailleurs : la psychologie historique de Jean-Pierre Vernant, mais aussi le modèle durkheimien du droit de Louis Gernet. L'effet «-logie », dans l'idée même d'archéologie et de généalogie, ne serait alors qu'une stratégie de plus, pour la philosophie, d'assurer sa position régalienne sur les sciences sociales. Mais comme je l'indique à la page $338 \mathrm{du}$ livre, il s'agit moins, avec la généalogie, d'annexer les sciences sociales ou de privilégier l'une d'entre elles pour y puiser ses modèles d'intelligibilité, que de se connecter "à une pluralité de disciplines, sans jamais se confondre avec aucune d'elles ». Cette connexion sans annexion ni exclusivisme méthodologique amène Foucault à décliner sous diverses formes son programme de recherches concernant les pratiques de vérité : histoire, géographie, ethnologie de la vérité. La philosophie est contrainte de penser aux frontières, car elle ne suffira pas à elle seule à répondre à " l'étonnement épistémique » qui la motive quant au fait qu'il y $a$ de la vérité, sous des formes multiples et très éclatées.

Comme le souligne Chiara Collamati, j'ai bien cherché à montrer la nécessité d'une " altération réciproque » entre la philosophie et les sciences sociales, en proposant de lire en ce sens la proposition sartrienne de pratiquer la philosophie avec des «disciplines auxiliaires ». Il importe, pour Sartre, que

${ }^{1}$ J. Derrida, Théorie et pratique, op. cit, p. 69. 
cette auxiliarité entre les savoirs soit réciproque et non unilatérale : on n'a pas affaire à un philosophe acceptant de se frotter aux sciences sociales, mais à un philosophe soucieux de restituer des formes situées d'intelligibilité pratique (l'intelligibilité d'une histoire en cours), dans une tension toujours relancée avec un horizon totalisant, visant l'intelligibilité de l'Histoire. La raison dialectique, logique de la pratique en situation, n'est possible que de façon locale, constamment interrompue, brisée ou déviée par les contre-finalités qu'elle engendre. Pour rendre compte de cette intelligibilité située des pratiques, les sciences sociales ne sont pas qu'un simple décor permettant au philosophe de jouer comme il l'entend avec les concepts. Elles sont l'occasion d'une mise à l'épreuve de sa propre posture, et d'une réinvention conceptuelle permanente. C'est en cela, comme je le suggère dans la conclusion, que l'on peut envisager, entre Foucault et Sartre, une pratique de la philosophie comme « histoire conceptuelle », procédant à une recollection du sens à même les pratiques. Cette recollection, c'est aussi et d'abord l'affaire des sciences sociales elles-mêmes, qui usent pour cela de protocoles appropriés (enquêtes quantitatives et qualitatives, administration de la preuve). Je défends alors l'idée que la posture commune à Foucault et à Sartre est celle d'une philosophie pratique des sciences sociales. Je propose de lire la Critique de la raison dialectique en ce sens. Pour répondre à la première question posée par Chiara Collamati, je dirais que les odyssées qui y sont déployées, « de la praxis individuelle au pratico-inerte », « du groupe à l'Histoire », peuvent être lues comme une manière de réinventer, en les altérant, l'existentialisme et la philosophie politique en les mettant à l'épreuve des sciences sociales: l'anthropologie structurale (Lévi-Strauss), les Annales (Braudel), l'historiographie de la Révolution française (Georges Lefebvre, Daniel Guérin), le culturalisme américain (Kardiner), etc. Ma proposition de lire Sartre et Foucault comme des « philosopheshistoriens » participe de cette exigence d'altération réciproque des discours : revendiquant explicitement l'un et l'autre l'héritage du Marx historien du 18 Brumaire et des Luttes de classes en France, Foucault et Sartre élaborent un modèle d'intelligibilité stratégique des pratiques à partir de l'archive des luttes ${ }^{1}$. Il s'agit pour les deux auteurs de privilégier dans le corpus marxien un régime de discours résolument critique - celui de l'histoire du présent et de l'enquête - en le faisant jouer contre d'autres régimes de discours (discours scientifique, discours prophétique, discours officiel du Parti), dans lesquels le marxisme s'est sclérosé lui-même, en refusant précisément de jouer le jeu de

\footnotetext{
${ }^{1}$ H. Oulc'hen, «L'Intelligibilité des luttes. Foucault et Sartre lecteurs des enquêtes historiques de Marx », dans C. Laval, L. Paltrinieri et F. Taylan [dir.], Marx \& Foucault. Lectures, usages, confrontations, Paris, La Découverte, 2015, p. 113-128.
} 
l'altération réciproque.

J'en viens, pour terminer cette réflexion sur la crise de frontières entre philosophies et sciences sociales induite par le problème de la pratique, à l'absence relative de Bourdieu dans le livre, pointée par Gildas Salmon. Ce n'est aucunement un oubli de ma part. Dans ma thèse de doctorat, dont l'ouvrage constitue une version remaniée et abrégée, Bourdieu occupait une place déterminante, dont on trouve encore des traces dans le livre. J'en relèverai deux. D'une part, Bourdieu fournit un modèle d'intelligibilité de la pratique que l'on trouve déjà à l'œuvre chez Sartre : celui de l'hystérésis ou, pour reprendre l'expression de Sartre, de la «désadaptation dans l'adaptation »'. Loin de se ramener à un ajustement mécanique aux normes, la pratique est avant tout l'expérience d'un décalage, d'un retard constitutif (et non accidentel) entre les dispositions agentives et les normes sociales. Les pratiques sont grevées d'une profonde discontinuité entre les habitus et les contraintes sociales ${ }^{2}$. Les approches scolastiques de la pratique s'efforcent de réduire cette douloureuse discontinuité, en la ramenant soit à l'autosuffisance des structures et des causes sociales (modèle objectiviste), soit à la force de la volonté d'acteurs rationnels, censés rejoindre sans heurts ni décalages la rationalité du monde social. La pratique comprise ainsi comme désadaptation constitutive montre la prégnance d'un certain legs sartrien chez Bourdieu. J'ai analysé ailleurs ce legs en montrant ce que les premières enquêtes de terrain de Bourdieu (en Kabylie et en Béarn) doivent au modèle de l'action et de l'attitude magique développé par Sartre dans l'Esquisse d'une théorie des émotions ${ }^{3}$. Comme je m'en explique dans la conclusion du livre, l'hystérésis constitue l'un des meilleurs « révélateurs » de la pratique et de sa logique. C'est à ce titre que je la tiens pour la notion clé permettant d'articuler la philosophie et les sciences sociales dans la perspective d'une altération réciproque. Je m'accorde tout à fait avec Chiara Collamati pour élargir l'usage de la notion d'hystérésis à la question de l'intelligibilité de l'histoire - l'intelligibilité des ensembles pratiques conduisant d'ailleurs, dans l'économie de la Critique de la Raison dialectique, à la question de l'intelligibilité de l'histoire qui, on le sait, fait l'objet du tome II. Dans mon ouvrage Sartre et le colonialisme. La critique d'un système, j'avais

\footnotetext{
${ }^{1}$ Sur ce point, cf. C. Gautier, La Force du social. Enquête philosophique sur la sociologie des pratiques de Pierre Bourdieu, Paris, Cerf, 2012.

${ }^{2} \mathrm{H}$. Oulc'hen, «L'Émotion dans la pratique. De Sartre à Bourdieu », dans F. Caeymaex, G. Cormann et V. de Coorebyter [dir.], Études sartriennes. — Vol. 17-18: Repenser l'Esquisse d'une théorie des émotions, Bruxelles, Ousia, 2014, p. 169-198. ${ }^{3}$ Je reprends bien sûr la fameuse formule forgée par Ernst Bloch dans Héritage de ce temps.
} 
déjà en vue cet usage élargi à la temporalité historique de l'hystérésis, puisqu'il s'agissait, à travers l'histoire du colonialisme algérien, de rendre compte du conflit entre deux histoires non-contemporaines ${ }^{1}$ - celle des colons, celle des colonisés - dans un dialogue critique avec, entre autres, les enquêtes de Bourdieu sur les sous-prolétaires algériens dans l'immédiat après-guerre ${ }^{2}$.

La deuxième trace de Bourdieu dans le livre concerne paradoxalement la « scène primitive » du problème de la pratique que j'examine à partir de la lecture foucaldienne de la Lettre VII de Platon dans Le Gouvernement de soi et des autres. Ce que Foucault fait ressortir de cette lettre, c'est une impitoyable critique de la posture scolastique en philosophie, par celui qui passe pour le plus scolastique des philosophes. Le modèle de l'Eros philosophe est supplanté par le modèle de l'ergon, où la philosophie se définit comme tâche pratique. Cette trace bourdieusienne dans la lecture de Platon par Foucault nous amène également à questionner la politique de la vérité du sociologue. Dans Le Sens pratique, Bourdieu définit sa démarche comme un mode de pensée qui permet de surmonter la "distance distante qui institue la pratique comme un objet, posé devant l'observateur comme un corps étranger », en donnant la possibilité de «se faire le sujet théorique de la pratique des autres ou de la sienne propre, mais tout autrement que ne le croient les chantres du vécu ${ }^{3}$. Se faire le sujet de la pratique des autres qu'on étudie et se faire le sujet de sa propre pratique, c'est tout un : cette subjectivation s'opère d'abord dans la pensée, dans une pratique de la théorie qui renvoie dos à dos le mythe d'une « distance distante » qui ne serait que pure théorie, et le mythe d'une pratique qui ne serait que pure proximité sans distance, rivée au vécu. Dans le texte intitulé " Comprendre » et publié dans La Misère du monde, Bourdieu assume ce jeu de l'altération réciproque des frontières entre disciplines en décrivant l'enquête sociologique comme un exercice spirituel, à la manière de l'ascèse stoïcienne..

\section{La vérité en pratiques}

Ces réflexions sur la politique de vérité du sociologue m'amènent au troisième thème que les questions qui m'ont été posées ont permis de faire apparaître :

\footnotetext{
${ }^{1}$ Cf. H. Oulc'hen, Sartre et le colonialisme. La critique d'un système, Baye, La Digitale, 2015. Je mobilise également dans le livre des références à Aron, Rancière et Balibar, comme contrepoints critiques aux thèses de Sartre.

${ }^{2}$ P. Bourdieu, Le Sens pratique, Paris, Minuit, 1980, p. 245-246.

${ }^{3}$ Je renvoie aux pages 200-202 du livre sur ce point.
} 
celui d'un déplacement de l'axe théorie/pratique à un axe pratique/vérité. Ce déplacement est surtout patent chez Foucault, dans la distance qu'il a prise visà-vis d'Althusser, l'amenant à récuser l'opposition théorie/idéologie comme non pertinente pour ressaisir l'intelligibilité des pratiques. On voit ce déplacement à l'œuvre également dans le parcours de Foucault lui-même, depuis l'archéologie des pratiques discursives à la généalogie des pratiques (ou "régimes ») de vérité, impliquant des modes de subjectivation.

Aborder la multiplicité des régimes de vérité immanents aux pratiques impliquait une confrontation entre Foucault et Sartre sur le type de fiction qu'ils ont institués à l'égard de l'histoire de la vérité. D'où la lecture croisée que j'ai proposée des cours de Foucault avec La Légende de la vérité de Sartre. La stratégie est risquée car, comme le suggère Chiara Collamati, la généalogiefiction foucaldienne et l'histoire-légende sartrienne ne risquent-elles pas de basculer dans la "prolifération des narrations mythopoïétiques »? C'est très exactement le nerf de la critique que Carlo Ginzburg adresse à Foucault, et plus largement, aux tenants de ce qu'il appelle le «rhetorical turn » en sciences sociales ${ }^{1}$. Pour répondre à Chiara Collamati, je dirais que Foucault comme Sartre parviennent à éviter cet écueil d'un émiettement ou d'une relativisation de la vérité sous l'effet de la prolifération des narrations, dans la mesure où la vérité est, chez eux, inséparable d'un sol raboteux — je mobilise à dessein cette expression wittgensteinienne dans le livre - qui n'est autre que celui des pratiques. La pratique constitue donc le garde-fou ultime qui empêche de fictionnaliser complètement la vérité. À l'instar de Juliette Simont dans son article sur Vérité et Existence, «Les fables du vrai $»^{2}$, je propose de lire La Légende de la vérité, texte de jeunesse peu connu ${ }^{3}$, comme l'esquisse d'une théorie de la vérité comme pratique, à l'état pratique.

Cette question de l'immanence de la vérité aux pratiques m'amène ensuite à éclaircir une difficulté pointée par Chiara Collamati sur le statut du concept chez Sartre. J'ai entretenu une ambiguïté à certains moments du livre concernant l'opposition entre d'un côté un Sartre, philosophe de la « notion », et de l'autre, un Foucault et un Althusser philosophes du « concept». J'ai cer-

${ }^{1}$ J. Simont, «Les Fables du vrai (à propos de Vérité et existence) », Les Temps Modernes n531-533 : Témoins de Sartre, Paris, Gallimard, 1990, vol. 1, p. 188-233.

2 Bien que commenté par d'éminents lecteurs de Sartre auxquels je me réfère: V. de Correbyter, Sartre avant la phénoménologie, Bruxelles, Ousia, 2005; E. Barot, «Aux racines de l'idéologie », dans Id. [dir.], Sartre et le marxisme, Paris, La Dispute, 2011, p. $253-284$.

${ }^{3}$ T. W. Adorno, Dialectique négative [1966], trad. fr. par le groupe de traduction du Collège de philosophie, Paris, Payot, 2003, p. 22-23. 
tainement exagéré cette opposition forgée par Sartre dans un contexte polémique - celui de la réponse aux structuralistes parue dans L'Arc en 1966. Ce faisant, j'ai sans doute reconduit une des coordonnées de la querelle que je m'efforçais par ailleurs de dépasser. Ce que Sartre entend par «notion », ce n'est en fait rien d'autre que du concept à l'état pratique, passible d'une dialectique locale en prise sur les singularités matérielles des situations. Pour reprendre les termes d'Adorno, je dirais que ce qu'il faut entendre derrière cet usage sartrien du terme de " notion », c'est une volonté de « désensorcellement du concept » : «La réflexion philosophique s'assure du non-conceptuel dans le concept» de sorte que " changer cette orientation de la conceptualité, la tourner vers le non-identique, c'est là la charnière d'une dialectique ${ }^{1}$. Mais en aucun cas, le désensorcellement n'appelle un abandon du concept : il appelle simplement à reconnaître l'irréductibilité de ce que Sartre appelle dans Qu'est-ce que la subjectivité ?, Questions de méthode et L'Idiot de la famille, la part du « non-savoir » dans le savoir. Ce non-savoir vécu n'en est pas moins pleinement rationnel : il est la condition de possibilité de la rationalité dialectique, et de la mise en intelligibilité située des pratiques. Cela me permet de répondre à la question de Soheil Ghanbari sur le rapport de Sartre à Hegel : loin de reconduire une pensée finie d'entendement séparateur, l'intelligibilité par «notions » dialectise le savoir en le mettant perpétuellement à l'épreuve du socle pratique et muet de non-savoir d'où il procède. La réalité incarnée et singularisée d'une lutte à multiples épicentres vient trouer la continuité du cours de l'Histoire en interrompant son cours homogène et linéaire. C'est pourquoi l'intelligibilité d'une lutte déborde le cadre formel des concepts a priori, comme le montre Sartre dans l'exemple du match de boxe. Pour reprendre une terminologie empruntée à Ernst Bloch, je dirais que seul un concept « in statu nascendi » permet de saisir une praxis en cours ${ }^{2}$. Les notions sartriennes, ce sont de tels concepts « in statu nascendi ». J'ai esquissé en ce sens quelques pistes de confrontation entre la dialectique sartrienne et la dialectique de la praxis déployée par Ernst Bloch dans son ultime ouvrage, Experimentum mundi : à l'instar du non-savoir sartrien, « l'obscurité de l'instant qui se vit », chez Bloch, est déjà une saisie intuitive, mais dialectiquement rationnelle, des contradictions du réel. Elle correspond à la première élaboration d'un « front »,

\footnotetext{
${ }^{1}$ E. Bloch, Experimentum Mundi. Question, catégorie de l'élaboration, praxis [1975], Paris, Payot, p. 38.

${ }^{2}$ Je développe ce point dans un texte à paraître : H. Oulc'hen, « Il sogetto utopico della storia. Programma, impulso, voluntà », dans C. Collamati et M. Farnesi Camellone [dir.], Lo Spirito dell'utopia un secolo dopo. Temporalità e politica in Ernst Bloch, Quodlibet Macereta, à paraître.
} 
où le monde se donne comme enjeu de luttes et de transformations, plutôt que comme objet de contemplation ${ }^{1}$.

\section{Pratiques, structures, institutions}

La question que posent Ferhat Taylan et Chiara Collamati sur la portée transformatrice, et par là-même politique, des pratiques du point de vue de leur institution m'amène à recontextualiser le propos de mon ouvrage dans un contexte plus large, débordant le cadre que je me suis donné au départ de l'articulation des pratiques et des structures. La séquence intellectuelle que j'ai étudiée est en effet fortement marquée par le problème de l'institution. Mon point de départ althussérien m'a amené à privilégier l'axe structure/pratiques plutôt que l'axe institution/pratiques, mais il est certain que ce deuxième axe, loin d'être un point aveugle de mon travail, en constitue au contraire une occasion privilégiée de prolongement et de reformulation. Pour le dire dans les termes de Balibar que j'ai mobilisés dans l'esquisse de cartographie des concepts de la pratique au début du livre, l'axe structure/pratiques renvoie à un concept matérialiste de la politique comme transformation, sur fond de conditions structurelles et conjoncturelles. L'autre de la structure, c'est la conjoncture, aléatoire et contingente. C'est ainsi que la causalité structurale mise en œuvre par Althusser pour faire droit à la complexité surdéterminée du réel, est à comprendre en même temps, du point de vue de la pratique politique - celle du Prince machiavélien par exemple - comme une causalité aléatoire. Dans ce dispositif théorique où la pratique politique est à venir, appelée à combler un vide, il n'y a guère de place pour une institutionnalisation transformatrice et positive des pratiques.

Chez Althusser, l'institutionnalisation des pratiques est plutôt pensée sur un mode reproductif, celui des appareils idéologiques, où les actes des individus interpellés sont enchâssés dans des pratiques, elles-mêmes enchâssées dans des rites. La question est alors de savoir quelle marge l'institution-appareil laisse-t-elle aux "mauvais sujets » qui ne se laissent pas interpeller. C'est sur ce mode soustractif qu'Althusser envisage un rapport critique à l'institution, y compris quand il s'agit de la pratique théorique, qu'il s'efforce de

\footnotetext{
${ }^{1}$ Je mentionnerai, pêle-mêle, le texte phare de Deleuze, «Instincts et institutions »; les travaux de Lefort et de Castoriadis pendant et après Socialisme ou barbarie ; la psychothérapie institutionnelle (Oury, Guattari, Deligny) ; l'antipsychiatrie (Laing, Cooper, Basaglia) ; l'analyse institutionnelle (Lourau, Lapassade) ; ou encore la réception de Goffman en France (Isaac Joseph).
} 
soustraire au contrôle du Parti. Mais Althusser ne fait qu'esquisser ici un programme de recherches, que d'autres se chargeront de poursuivre en partie contre lui, dans une direction relevant davantage de l'analyse institutionnelle que de l'analyse idéologique. Comme exemple de prolongement et de reformulation de la critique althussérienne de l'institution, je mentionnerai le travail de Fernand Deligny, en particulier son ouvrage Le Croire et le craindre (1978), qui forge un concept original d'humain : "L'Humain sans sujet ni fin(s), tout comme l'histoire ${ }^{1}$. Cette invocation d'un " humain » sans sujet ni fin, qui semble à première vue peu compatible avec l'antihumanisme théorique althussérien, est pourtant le pendant de l'histoire comme procès sans sujet. C'est l'humain « de nature », primordial, qui subsiste sous le sujet de l'interpellation $^{2}$. Le couple institution/pratiques recouperait alors, dans la perspective de Deligny, la distinction entre un faire indexé sur des fins prédéfinies, et un agir intransitif, infinitif. Ou encore, la distinction entre ce qu'il appelle précisément l'institution, c'est-à-dire le fait, pour un bon sujet, de «trouver une place », et la tentative, c'est-à-dire le fait pour un mauvais sujet de « trouver de la place » en esquivant les places institutionnelles.

J'ai suggéré dans une section du chapitre 2 de l'ouvrage intitulée «L'idéologie en pratiques » que le structuralisme de la pratique althussérien peut se décliner de deux manières : la voie par le haut d'un fonctionnalisme de la reproduction, où l'institution est conçue comme pouvoir localisé (par un appareil), possédé (par une classe dirigeante), et subordonné à une infrastructure matérielle ; et la voie par le bas, non développée par Althusser, d'une lecture micro-analytique des pratiques. C'est cette deuxième voie, qu'explore Foucault dans sa « microphysique » des pouvoirs disciplinaires, qui me semble la plus à même de penser la répétabilité des pratiques dans un dispositif donné, sans reconduire pour autant à ce que Bourdieu appellerait un « fonctionnalisme du pire ». Faut-il alors abandonner la notion d'institution ? Dans la leçon d'ouverture du Pouvoir psychiatrique, Foucault dit regretter s'être servi cette notion qui n'est qu'une « serrure rouillée » avec laquelle « on ne peut pas avancer beaucoup $»^{3}$. Il suspecte le concept d'institution de présupposer des régularités

\footnotetext{
${ }^{1}$ F. Deligny, Euvres, Paris, L’Arachnéen, 2017, p. 1150.

${ }^{2}$ Sur le rapport Deligny/Althusser, $c f$. P. Macherey, Le Sujet des normes, Paris, Amsterdam, 2014, p. 102-131.

${ }^{3}$ F. Deligny, Euvres, op. cit., p. 1118. Je renvoie également au début du Croire et le craindre, à consonances fortement althussériennes, où Deligny décrit sa première rentrée scolaire déjà comme une tentative de trouver de la place, et non de trouver une place prédéfinie dans l'institution : « Il fallait que je rentre là-dedans. C'est sûr que je suis rentré, mais quelque chose est resté dehors ; le sujet est rentré ; le bon sujet ou le mauvais, ça dépend des moments. Le sujet est rentré à l'école, comme il faut, puisqu'il
} 
entre des individus et des collectifs déjà donnés, régularités dont se repaissent la plupart des discours psychologiques et sociologiques. Focalisés sur la consistance sui generis du social, ces discours restent aveugles aux « déséquilibres du pouvoir », dont participent « les réseaux, les courants, les relais, les points d'appui, les différences de potentiel qui caractérisent une forme de pouvoir $»^{1}$. Ainsi, loin d'être donnés au départ, l'individu, le collectif et, partant, l'institution, sont les effets de ces déséquilibres du pouvoir. Il s'agit moins de récuser l'institution en bloc que d'expliciter des processus d'institutionnalisation dont participe la fabrication des corps dociles et des âmes obéissantes. En ce sens, l'usage que Foucault fait du concept d'institution est semblable à celui qu'il fait du concept d'État, lorsqu'il propose de ressaisir des processus d'étatisation des relations mobiles de pouvoir ${ }^{2}$.

Le couple institution/pratiques n'est donc légitime, chez Foucault, que s'il permet de faire droit à la réalité matérielle des déséquilibres du pouvoir, qui empêchent toute autorégulation, toute clôture réifiante, des dispositifs de pouvoir. L'institutionnalisation des pratiques n'est pas uniquement du côté des disciplines, c'est-à-dire des pratiques que l'on répète docilement. Elle recouvre aussi ce que Ferhat Taylan appelle très justement les «pratiques que l'on souhaiterait répéter ", à savoir les modes multiples de subjectivation, de rapport à soi, que les sujets inventent, instituent dans leur rapport à la vérité. C'est précisément ce que j'ai appelé « l'horizon éthopoiétique des pratiques ». Chez les lecteurs du dernier Foucault, cet horizon éthopoiétique a souvent été dépolitisé dans le sens d'une célébration dandy ou new age de l'esthétique de l'existence. Or Foucault donne au contraire des outils précieux pour penser une politisation du soi, qui ne part pas de rien mais qui s'appuie sur les « schémas » que l'individu « trouve dans sa culture et qui lui sont proposés, suggérés, imposés $»^{3}$. À travers les formes historiques de l'aveu et de la libre parole (parrêsia), il s'agit bien d'instituer, en médiatisant les schèmes de la pratique disponibles dans une culture donnée, des pratiques de soi qui impliquent une certaine reconfiguration de l'intersubjectivité : toute institution d'un dire-vrai suppose un pour-autrui, comme je l'ai montré dans la section du chapitre $3 \mathrm{du}$ livre consacrée à la lecture foucaldienne de la Lettre VII de Platon.

le fallait, mais quelque chose est resté dehors, quelque chose de très vivace, comme du chiendent » (F. Deligny, Croire et le craindre, dans EEuvres, op. cit., p. 1092).

${ }^{1}$ M. Foucault, Le Pouvoir psychiatrique. Cours au Collège de France, 1973-1974, Paris, Gallimard-Seuil, 2003, p. 15.

${ }^{2}$ M. Foucault, Le Pouvoir psychiatrique, op. cit., p. 16.

${ }^{3}$ Sur ce point, cf. A. Skornicki, La Grande soif de l'État. Michel Foucault avec les sciences sociales, Paris, Les Prairies ordinaires, 2015. 
Quelques remarques, pour finir, sur l'épineuse question soulevée par Ferhat Taylan de l'institution du commun. Il est certain que l'invention des pratiques de soi que Foucault analyse à travers les formes du dire-vrai peine à dépasser le niveau de l'intersubjectif - que Ferhat Taylan distingue du collectif. Ce point d'achoppement sur la question du commun est peut-être l'indice d'un « aristocratisme paradoxal » chez Foucault, comme le souligne Balibar dans son essai récemment publié, Libre parole. Le courage et le scandale de la vérité, « exercice risqué d'un dire-vrai qui insiste en excès par rapport à l'institution ", serait la condition paradoxale (en ce qu'il implique un ascendant éthique) d'une " certaine institution de l'égalité », ramenant la démocratie à son principe ${ }^{1}$. Instituer l'égalité comme droit égal à parler (isegoria) et à gouverner (isonomia) implique une contre-conduite que l'on peut comprendre en même temps comme une contre-institution ${ }^{2}$. Or les contre-conduites ne permettent pas d'instituer du commun au sens fort du terme, impliquant une transformation globale des relations de pouvoir. Tout au plus permettent-elles d'instituer des collectifs spécifiques - tels que le GIP — relayés par des intellectuels eux-mêmes spécifiques ${ }^{3}$. Foucault, on le sait, a voulu en découdre avec le modèle institutionnellement surdéterminé du tribunal et de l'intellectuel comme juge redresseur de torts. A contrario, c'est ce modèle du tribunal populaire que Sartre a réinvesti contre les institutions bourgeoises de la justice, notamment avec son réquisitoire lors de l'accident minier survenu à HéninLiétard en $1970^{4}$. Il s'agit pour Sartre de dés-instituer l'institution en la réinstituant dans une praxis commune. Ce qui implique, ce que ne fait pas Foucault, de dialectiser l'institution.

Il me parait fructueux en ce sens de mobiliser le lexique de l'analyse institutionnelle, et en particulier la distinction opérée par René Lourau entre l'instituant et l'institué, qui recoupe la distinction sartrienne entre la dialectique constituante et la dialectique constituée ${ }^{5}$. L'institué désigne, au sens large, les strates d'inertie des groupes, qui ne se réduisent pas au seul cadre de

\footnotetext{
${ }^{1}$ M. Foucault, «L’Éthique du souci de soi comme pratique de la liberté » [1984], dans Dits et écrits. Tome II : 1976-1984, Paris, Gallimard, 2001, p. 1538.

${ }^{2}$ Selon l'expression de Philippe Descola dans Par-delà nature et culture, Paris, Gallimard, 2005.

${ }^{3}$ Cf. É. Balibar, Libre parole, Paris, Galilée, 2018, p. 118-119.

${ }^{4}$ Balibar, dans Libre parole, op. cit., p. 119, parle quant à lui d'une « figure de "contredémocratie" qui représente pourtant l'action démocratique par excellence».

${ }^{5}$ Les figures grecques du parrèsiaste constituent une mise en abîme de l'intellectuel spécifique, comme j'ai essayé de le montrer ailleurs : $c f$. H. Oulc’hen, « La Politique de la vérité de l'intellectuel. Entre Foucault et Sartre », dans Id. [dir.], Usages de Foucault, Paris, PUF, 2014, p. 293-318.
} 
la souveraineté comme pétrification du groupe. L'institué, praxis et chose à la fois, est tout aussi bien : $1^{\circ}$ le savoir accumulé qui définit l'esprit objectif d'une époque (ou ce que Sartre appelle « la culture comme pratico-inerte ${ }^{1}$ ) ; $2^{\circ}$ les impératifs moraux qui définissent les choses « à faire » à une époque donnée ; $3^{\circ}$ enfin, de façon plus tragique, «le choix inerte et stratifié qu'une société fait de ses morts $»^{2}$ dans le cadre de la rareté et de la division du travail - cette « thanapolitique » pouvant être portée par un dispositif de souveraineté. L'institué assure donc la répétabilité des pratiques, en définissant pour les individus un espace de possibles culturels, moraux et même vitaux, l'avenir étant vécu, pour certains, comme irrémédiablement «barré » ${ }^{3}$. L'institué sérialise les individus. Que fait, par opposition, l'instituant ? Je me réfère à la définition riche qu'en donne Olivier Quintyn (dans la reprise qu'il fait du doublet de Lourau pour sa théorie de l'avant-garde) : «L'instituant bouscule, sous le coup de la critique, les pôles institués, de façon à les déplacer et à redéployer autrement leurs liens, jusqu'à les abroger et à les reconstruire intégralement en situation d'urgence »". De même que l'institué ne s'épuise pas dans la forme pétrifiante de la souveraineté, l'instituant ne se ramène pas au seul «pouvoir constituant » des multitudes. Il désigne tous les modes par lesquels la praxis, individuelle ou commune, se suscite elle-même pour réinventer les réciprocités pratiques instituées, cette réinvention pouvant aller du simple déplacement symbolique sans grandes conséquences, jusqu'à l'abrogation et la reconstruction intégrale dans des situations d'urgence et de pression d'une praxis adverse. Véritable expérimentation pratique, l'instituant est donc à comprendre comme un réinstituant, puisqu'il fait fond sur un institué duquel il ne saurait se soustraire.

On peut ainsi proposer, à partir de Sartre, une lecture dialectique de l'institution, permettant de dépasser le clivage de l'intersubjectif et du commun qui constitue le point d'achoppement des derniers cours de Foucault sur la parrêsia. Cela me permet de répondre à la question posée par Chiara Collamati sur la place du normatif dans le cadre d'une généalogie du pré-droit et des pratiques archaïques de vérité : la mise en perspective des formes instituées modernes du droit par rapport à une strate archaïque où le droit est pensé en

\footnotetext{
${ }^{1}$ J.-P. Sartre, Situations, VIII. Autour de 68, Paris, Gallimard, 1972, p. 319-331. Mentionnons aussi la première partie de ce même volume (pp. 9-124), qui regroupe tous les textes que Sartre a consacrés au Vietnam et au Tribunal Russell.

${ }^{2}$ On sait que l'analyse institutionnelle s'est largement inspirée de la Critique de la Raison dialectique pour thématiser les groupes-sujets et les groupes-objets.

${ }^{3}$ J.-P. Sartre, L'Idiot de la famille, Paris, Gallimard, 1972, tome 3, p. 43.

${ }^{4}$ J.-P. Sartre, Critique de la Raison dialectique. Tome I : Théorie des ensembles pratiques [1960], Paris, Gallimard, 1985, p. 241.
} 
continuité avec la lutte apporte une lumière pour dés-instituer l'institué et, par là-même, le réinstituer autrement. 